\section{International Scientific Journal Theoretical \& Applied Science}

\author{
p-ISSN: 2308-4944 (print) e-ISSN: 2409-0085 (online) \\ Year: $2016 \quad$ Issue: $5 \quad$ Volume: 37 \\ Published: $30.05 .2016 \quad \underline{\text { http://T-Science.org }}$
}

SECTION 2. Applied mathematics. Mathematical modeling.

\section{X.A. Mamadaliyev,}

Senior Researcher, Centre for the development of software and hardware-program complexes at TUIT,

Tashkent, Uzbekistan

husniddin_m1@bk.ru

I.Q. Khujayev, Leading Researcher, Centre for the development of software and hardware-program complexes at TUIT,

Tashkent, Uzbekistan i_k_hujayev@mail.ru

\title{
SEALING WAVE PROPAGATION, DUE TO THE DECELERATION OF FLUID FLOW IN AN INCLINED PIPELINE
}

Abstract: In the article, an analytical solution of the problem of shock wave propagation in an inclined pipeline formed by stoppage of fluid flow is obtained. A linearized system of pipeline transportation droplet equations is involved for modeling the process. The system of equations is brought to the individual equations for pressure and flow rate in the form of incomplete telegraph equations. These equations are solved by the method of separating variables.

On the basis of the solution a computational experiment is carried out for different lengths and slopes of the site. Depending on the slope of the pipeline the areas where high pressure is expected are identified.

Key words: pipeline transportation, mathematical modeling, shock wave, the slope of the pipeline, velocity, pressure, computing experiment.

Language: Russian

Citation: Mamadaliyev XA, Khujayev IQ (2016) SEALING WAVE PROPAGATION, DUE TO THE DECELERATION OF FLUID FLOW IN AN INCLINED PIPELINE. ISJ Theoretical \& Applied Science, 05 (37): 105-114.
Soi: http://s-o-i.org/1.1/TAS-05-37-20
Doi: crossef http://dx.doi.org/10.15863/TAS.2016.05.37.20

\section{РАСПРОСТРАНЕНИЕ ВОЛНЫ УПЛОТНЕНИЯ, ВЫЗВАННОЙ ТОРМОЖЕНИЕМ ЖИДКОСТИ В НАКЛОННОМ ТРУБОПРОВОДЕ}

Аннотация: В статье получено аналитическое решение задачи о распространении ударной волны в наклонном трубопроводе, образованной торможением жидкости. Для моделирования прочесса привлечена линеаризованная система уравнений трубопроводного транспорта капельной жидкости. Система уравнений приведена к отдельным уравнениям относительно давления и скорости потока, имеющие вид неполных телеграфных уравнений. Полученные уравнения решены методом разделения переменных.

На основе полученного решения проведен вычислительный эксперимент для различных длин и уклона участка. В зависимости от уклона трубопровода определены участки, где ожидается высокое давление.

Ключевые слова: трубопроводный транспорт, математическое моделирование, скачок уплотнения, уклон трубопровода, скорость, давление, вычислительный эксперимент.

Введение. В стадиях предварительного расчета и проектирования сети трубопроводов пользуются нормативными документами, которые составлены на основе стабильных показателей течения в различных режимах. Но для исследования надежности их целесообразно обратиться к динамическим задачам, которые указывают их узкие места, связанные, например, обеспечением необходимым давлением и расходом в точке подключения потребителя или образованием и распространением волн высокого давления.
Разработаны и разрабатываются аналитические и численные методы решения задач о динамических процессах в сети трубопроводов.

В частности, многочисленные задачи о распространении волн возмущений давления и расхода и методов сглаживания их скачков рассмотрены в монографии И.А.Чарного [1]. В качестве примера использования классического метода разделения переменных автор обратился к сформулированной Н.Е.Жуковским задаче о распространении гидравлического удара в горизонтальном трубопроводе. Проведенные 


\begin{tabular}{|c|c|c|c|c|c|c|}
\hline Impact Factor: & $\begin{array}{l}\text { ISRA (India) } \\
\text { ISI (Dubai, UAF } \\
\text { GIF (Australia) } \\
\text { JIF }\end{array}$ & $\begin{array}{l}=1.344 \\
=0.829 \\
=0.564 \\
=1.500\end{array}$ & $\begin{array}{l}\text { SIS (USA) } \\
\text { PИНЦ (Russia) } \\
\text { ESJI (KZ) } \\
\text { SJIF (Morocco) }\end{array}$ & $\begin{array}{l}=0.912 \\
=0.234 \\
=1.042 \\
=2.031\end{array}$ & $\begin{array}{l}\text { ICV (Poland) } \\
\text { PIF (India) } \\
\text { IBI (India) }\end{array}$ & $\begin{array}{l}=6.630 \\
=1.940 \\
=4.260\end{array}$ \\
\hline
\end{tabular}

нами расчеты на основе данного решения привели к синусоидальным изменениям, которые весьма далеки от ожидаемой картины процесса.

В связи с этим в рамках данной работы обратились к задаче торможения жидкости, дополнительно учитывая исходное распределение давления согласно постоянному расходу жидкости и уклона оси трубопровода от горизонта. Согласно полученным результатам провели вычислительный эксперимент.

Постановка задачи. Для описания процесса распространения волны уплотнения, образованной торможением жидкости, пользовались системой квазиодномерных уравнений сохранения импульса и массы малосжимаемой жидкости по наклонному трубопроводу [1]

$$
\left\{\begin{array}{l}
-\frac{\partial p}{\partial x}=\rho\left(\frac{\partial w}{\partial t}+2 a w+g \sin \alpha\right), \\
-\frac{\partial p}{\partial t}=\rho c^{2} \frac{\partial w}{\partial x} .
\end{array}\right.
$$

Здесь переменные $p$ и $w$ представляют статическое давление и среднерасходнюю скорость жидкости в сечение $x$ в момент времени $t$. Параметр $c$ означает скорость распространения волн возмущения давления, вычисленная с учетом модулей упругости жидкости и материала трубы [2,3]; а в остальном жидкость считается несжимаемой ( $\rho \approx$ const $)$. Уклон трассы учитывается через параметр $\sin \alpha=\frac{d y}{d x}=$ const, где $y(x)-$ пьезометрическая высота оси трубопровода. Сила сопротивления трения определена коэффициентом $a=\lambda w_{*} /(4 D)=$ const, где $\lambda-$ коэффициент силы сопротивления, $w_{*}$ - характерная скорость для рассматриваемого процесса (или параметр осреднения по И.А.Чарному), $D$ - диаметр трубопровода. В уравнениях $g$ представляет ускорение силы гравитации.

Считаем, что до торможения жидкости трубопровод функционировал с постоянной скоростью $w_{0}$, а на входе в участок давление составляло $p_{00}$. Согласно этих данных начальными условиями для задачи служат

$$
\begin{aligned}
& w(x, 0)=w_{0}=\text { const }, \\
& p(x, 0)=p_{00}-2 a \rho w_{0} x-\rho g \sin \alpha x .
\end{aligned}
$$

Начиная с момента времени $t=0$ в конце трубопровода скорость из $w_{0}$ переходит к $A$

$$
w(l, t)=A,
$$

а на входе в участок значение давления осталось постоянной

$$
p(0, t)=p_{00},
$$

Требуется найти решения задачи относительно скорости потока $w(x, t)$ и давления $p(x, t)$.

Как видно из постановки задачи, в отличие от [1], учитываются исходное распределение давления и уклон трубопровода от горизонта.

Решение задачи. Введением новой искомой

$$
u(x, t)=w(x, t)+\frac{g \sin \alpha}{2 a}=w(x, t)+A_{1}
$$

из системы (1) исключим силу гравитации $\rho g \sin \alpha$ :

$$
\left\{\begin{array}{l}
-\frac{\partial p}{\partial x}=\rho\left(\frac{\partial u}{\partial t}+2 a u\right) \\
-\frac{\partial p}{\partial t}=\rho c^{2} \frac{\partial u}{\partial x}
\end{array}\right.
$$

Но она будет фигурировать в краевых условиях:

$$
\begin{aligned}
& u(x, 0)=w_{0}+A_{1}=u_{0}, \quad p(x, 0)=p_{00}-2 a \rho u_{0} x, \\
& p(0, t)=p_{00}, \quad u(l, t)=A+A_{1}=\text { const. }
\end{aligned}
$$

Из системы (5) и условий (6) составим отдельное уравнение относительно $u(x, t)$

$$
\frac{\partial^{2} u}{\partial t^{2}}+2 a \frac{\partial u}{\partial t}=c^{2} \frac{\partial^{2} u}{\partial x^{2}},
$$

и краевые условия:

$$
\left.\begin{array}{l}
u(x, 0)=u_{0}, \frac{\partial u(x, 0)}{\partial t}=0, \\
\frac{\partial u(0, t)}{\partial x}=0, \quad u(l, t)=A+A_{1} \cdot
\end{array}\right\}
$$

В (7) и (8) переходим к однородным граничным условиям [2], для чего вводится новая искомая

$$
V(x, t)=u(x, t)-\frac{A+A_{1}}{l^{2}} x^{2} .
$$

Краевые условия приобретают вид

$$
\left.\begin{array}{l}
V_{1}(x, 0)=u_{0}-\frac{A+A_{1}}{l^{2}} x^{2}, \quad \frac{\partial V_{1}(x, 0)}{\partial t}=0, \\
\frac{\partial V_{1}(0, t)}{\partial x}=0, \quad V_{1}(l, t)=0 .
\end{array}\right\}
$$

Уравнение становится неоднородным:

$$
\frac{\partial^{2} V}{\partial t^{2}}+2 a \frac{\partial V}{\partial t}-c^{2} \frac{\partial^{2} V}{\partial x^{2}}=\frac{2 c^{2}}{l^{2}}\left(A+A_{1}\right)
$$

Решение задачи (10)-(11) можно искать в виде суммы

$$
V(x, t)=V_{1}(x, t)+V_{2}(x, t),
$$

где $V_{1}(x, t) \quad-$ решение однородного уравнения

$$
\frac{\partial^{2} V_{1}}{\partial t^{2}}+2 a \frac{\partial V_{1}}{\partial t}-c^{2} \frac{\partial^{2} V_{1}}{\partial x^{2}}=0
$$




\begin{tabular}{l|lr|ll|ll} 
& ISRA (India) & $=\mathbf{1 . 3 4 4}$ & SIS (USA) & $=\mathbf{0 . 9 1 2}$ & ICV (Poland) & $\mathbf{= 6 . 6 3 0}$ \\
Impact Factor: & ISI (Dubai, UAE) $=\mathbf{0 . 8 2 9}$ & PUHL (Russia) $=\mathbf{0 . 2 3 4}$ & PIF (India) & $=\mathbf{1 . 9 4 0}$ \\
& GIF (Australia) & $=\mathbf{0 . 5 6 4}$ & ESJI (KZ) & $=\mathbf{1 . 0 4 2}$ & IBI (India) & $=\mathbf{4 . 2 6 0}$
\end{tabular}

при тех же краевых условиях, что и в (10), а $V_{2}(x, t)$ - решение неоднородного уравнения

$$
\frac{\partial^{2} V_{2}}{\partial t^{2}}+2 a \frac{\partial V_{2}}{\partial t}-c^{2} \frac{\partial^{2} V_{2}}{\partial x^{2}}=\frac{2 c^{2}}{l^{2}}\left(A+A_{1}\right)
$$

при нулевых краевых условиях.

Поиск $V_{1}(x, t)$ в виде ряда

$$
V_{1}(x, t)=\sum_{n=1}^{\infty} T_{n}(t) X_{n}(x)
$$

приводит к собственным функциям

$$
\begin{gathered}
X_{n}(x)=\sin \frac{2 n-1}{2} \frac{\pi(l-x)}{l}, \\
T_{n}(t)=e^{-a t}\left(\begin{array}{l}
C_{n} \operatorname{ch} \sqrt{\mathfrak{D}_{n}} t+D_{n} \operatorname{sh} \sqrt{\mathfrak{D}_{n}} t \quad n p u \mathfrak{D}_{n}>0, \\
D_{n} t+C_{n} \quad n p u \mathfrak{D}_{n}=0, \\
C_{n} \cos \sqrt{\left|\mathfrak{D}_{n}\right|} t+D_{n} \sin \sqrt{\left|\mathfrak{D}_{n}\right|} t n p u \mathfrak{D}_{n}<0 .
\end{array}\right),
\end{gathered}
$$

где $\mathfrak{D}_{n}=a^{2}-\left(\frac{2 n-1}{2} \frac{\pi c}{l}\right)^{2},\left\|X_{n}\right\|^{2}=l / 2$.

В отличие от [1] для $T_{n}(t)$ учитываются случаи $\mathfrak{D}_{n} \geq 0$. В самом деле, если например, принять $\lambda=0.02, w_{*}=10 \mathrm{M} / \mathrm{c}, D=0.2 \mathrm{M}, c=1200$ м/с и $l=10$ км, то имеем $\mathfrak{D}_{1}>0$. По этому не следует игнорировать случай $\mathfrak{D}_{n}=0$ [].

Реализация начальных условий приводит к решению

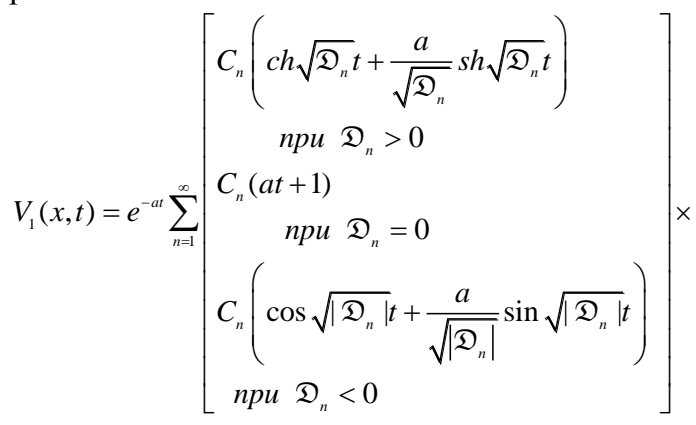$$
\times \sin \frac{2 n-1}{2} \frac{\pi(l-x)}{l} .
$$

где

$$
\begin{aligned}
C_{n}= & \frac{4}{(2 n-1) \pi} u_{0}-\left(\frac{4}{(2 n-1) \pi}-\frac{32}{(2 n-1)^{3} \pi^{3}}\right) \times \\
& \times\left(A+A_{1}\right) .
\end{aligned}
$$

Аналогичным образом получено решение неоднородного уравнения

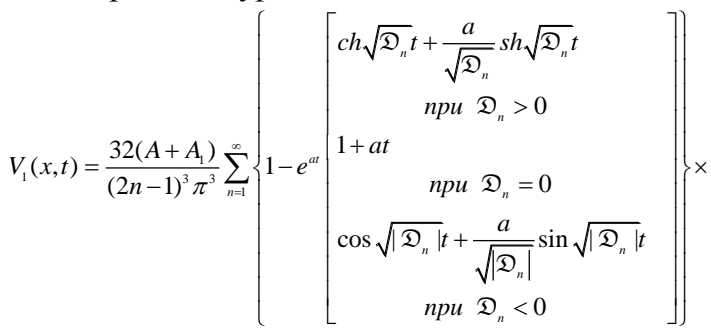

$$
\begin{aligned}
& \times \sin \frac{2 n-1}{2} \frac{\pi(l-x)}{l} \text {. }
\end{aligned}
$$

Суммируя $V_{1}(x, t)$ и $V_{2}(x, t)$ находим $V(x, t)$.

Обратные переходы к $u(x, t)$ согласно (9) и к $w(x, t) \quad$ и последующие упрощающие видоизменения приводят к конечному результату для динамической скорости

$$
w(x, t)=A+e^{-a t} \sum_{n=1}^{\infty} \frac{4\left(w_{0}-A\right)}{(2 n-1) \pi}\left[\begin{array}{cc}
c h \sqrt{\mathfrak{D}_{n}} t+\frac{a}{\sqrt{\mathfrak{D}_{n}}} s h \sqrt{\mathfrak{D}_{n}} t \\
n p u & \mathfrak{D}_{n}>0 \\
1+a t & n p u \mathfrak{D}_{n}=0 \\
\cos \sqrt{\left|\mathfrak{D}_{n}\right|} t+\frac{a}{\sqrt{\left|\mathfrak{D}_{n}\right|}} \sin \sqrt{\left|\mathfrak{D}_{n}\right|} \\
n p u \mathfrak{D}_{n}<0
\end{array}\right] \times
$$

Из различных вариантов решения задачи относительно давления только описываемый ниже вариант позволил получить адекватное решение.

Из системы (1) составили уравнение относительно давления

$$
\frac{\partial^{2} p}{\partial t^{2}}+2 a \frac{\partial p}{\partial t}-c^{2} \frac{\partial^{2} p}{\partial x^{2}}=0 .
$$

Краевыми условиями данного уравнения служили:

$$
\begin{aligned}
p(x, 0)= & p_{00}-2 a \rho w_{0} x-\rho g \sin \alpha x, \\
& \frac{\partial p(x, 0)}{\partial t}=0 . \\
p(0, t)=p_{00}, & \frac{\partial p(l, t)}{\partial x}=-\rho g \sin \alpha .
\end{aligned}
$$

Переход к однородным граничным условиям осуществляли заменой

$$
v(x, t)=p(x, t)-p_{00}+\rho g \sin \alpha x .
$$

Краевые условия для $v(x, t)$ составили

$$
\left.\begin{array}{l}
v(x, 0)=-2 a \rho w_{0} x, \quad \frac{\partial v(0, t)}{\partial t}=0, \\
v(0, t)=0, \quad \frac{\partial v(l, t)}{\partial x}=0 .
\end{array}\right\}
$$

В этом случае уравнение получилось однородным [4]:

$$
\frac{\partial^{2} v}{\partial t^{2}}+2 a \frac{\partial v}{\partial t}-c^{2} \frac{\partial^{2} v}{\partial x^{2}}=0
$$

При сохранении обозначения $\mathfrak{D}_{n}=a^{2}-\left(\frac{2 n-1}{2} \frac{\pi c}{l}\right)^{2}$ нами получено решение в виде 


\begin{tabular}{|c|c|c|c|c|c|c|}
\hline Impact Factor: & $\begin{array}{l}\text { ISRA (India) } \\
\text { ISI (Dubai, UAF } \\
\text { GIF (Australia) } \\
\text { JIF }\end{array}$ & $\begin{array}{l}=1.344 \\
=0.829 \\
=0.564 \\
=1.500\end{array}$ & $\begin{array}{l}\text { SIS (USA) } \\
\text { PИНЦ (Russia) } \\
\text { ESJI (KZ) } \\
\text { SJIF (Morocco) }\end{array}$ & $\begin{array}{l}=0.912 \\
=0.234 \\
=1.042 \\
=2.031\end{array}$ & $\begin{array}{l}\text { ICV (Poland) } \\
\text { PIF (India) } \\
\text { IBI (India) }\end{array}$ & $\begin{array}{l}=6.630 \\
=1.940 \\
=4.260\end{array}$ \\
\hline
\end{tabular}

$p(x, t)=p_{00}-\rho g \sin \alpha x+$

$+16 a \rho w_{0} l e^{-a t} \sum_{n=1}^{\infty} \frac{(-1)^{n}}{(2 n-1)^{2} \pi^{2}}\left(\begin{array}{c}\operatorname{ch} \sqrt{\mathfrak{D}_{n}} t+\frac{a}{\sqrt{\mathfrak{D}_{n}}} \operatorname{sh} \sqrt{\mathfrak{D}_{n}} t \\ n p u \mathfrak{D}_{n}>0 \\ 1+\text { at } n p u \\ \cos \sqrt{\left|\mathfrak{D}_{n}\right|} t+\frac{a}{\sqrt{\left|\mathfrak{D}_{n}\right|}} \sin \sqrt{\left|\mathfrak{D}_{n}\right|} \\ n p u \mathfrak{D}_{n}<0\end{array}\right) \times$

$\times \sin \frac{2 n-1}{2} \frac{\pi x}{l}$

Результаты

вычислительного

эксперимента. На основе приведенных выше решений разработана программа расчета в среде Delphi 7. Результаты расчета обрабатывались с помощью Microsoft Excel-приложения Windows.

Расчета проводились при $D=0.200 \quad \mathcal{M}$, $\lambda=0,018, \quad c=1200 \mathrm{M} / \mathrm{c}, \quad A=0.0 \mathrm{M} / \mathrm{c} \quad w_{*}=w_{0}=12.0$ $M / c$. Длину участка $l$ разделили на 50 равные части. Шаг по времени составил $l /(4 c)$ - одну четвертую часть времени пробега волны с одного конца участка до другого. Чтобы исключить визуальные осцилляции число разложений в вычислениях составило 500 членов. Для горизонтального трубопровода $(\sin \alpha=0)$ расчеты проводились для $l=10000,5000,2000$ и $1000 \mathrm{M}$.

На рис. 1 приведены графики скорости потока для времен $t=0, l /(4 c), \ldots, 2 l / c$.

Верхняя прямая соответствует исходному распределению скорости по длине участка. После торможения потока скорость убывает до нуля. В промежутке $t=(0 ; l / c)$ кривая скорости состоит из трех частей: не достигшая фронтом скачка часть, сам фронт скачка и участок следствия торможения. При $t=l / c$ скорость имеет монотонно убывающий график. С этого момента до $t=2 l / c$ график скорости получается перевернутый: сначала часть с меньшей скорости, потом скачок возрастания скорости и в конце - участок, график которого практически повторяет графика скорости при $t=l / c$. Дальше образуется следующая монотонная линия скорости, которая расположена намного ниже кривой скорости, полученной при $t=l / c$ и т.д.

Увеличение длины участка $(l \geq 10000$ м $)$ приводит к классическим графикам решения уравнения теплопроводность с тепловыми волнами.
На рис. 2 приведены графики избыточного давления $p-p 00$, соответствующие данным рис. 1.

Нижняя прямая линия соответствует исходному распределению $p-p 00$ на участке. C торможением жидкости образуется скачок давления, который перемещается против потока. Величина скачка убывает по времени и пространству. В промежутке $t=(0 ; l / c)$ графики, как и в случае скорости, состоят из трех частей: часть, до которой не дошел скачок уплотнения, сам скачок уплотнения и часть за скачком уплотнения.

С достижением левого конца участка скачок переворачивается и перемещается в обратное направление. Скачки постепенно убывают.

При $l \geq 10000 м$ и при $t \geq l / c$ скачки давления практически исчезают. Притом сохраняется тенденция знакопеременности графиков $p-p_{00}$, полученных при $n l / c$.

C сокращением длины участка $l$ становится ощутимым число отражений волны уплотнения с явными образованием огибающих кривых скорости и статического давления.

На рис. 3 и 4 приведены соответственно кривые скорости и давления шагом $l /(4 c)$ для пяти первых отрезков времени $2 l / c$.

Огибающих кривых скорости (в рамках визуализации) составляют сами кривые скорости, полученные при $t=0, l / c, 3 l / c, 5 l / c, \ldots$ При $t=0$ в качестве огибающей имеем $w(x, t)=w_{0}=$ const, $\quad$ при $\quad t=l / c \quad$ получим практически прямую линию с отрицательным угловым коэффициентом, при $t=3 l / c-$ угловой коэффициент положительны. То есть имеем (кроме первой) знакопеременный угловой коэффициент линий огибающих, стремящийся к нулю.

Огибающие кривых давления (рис. 4) представляют кривых давления, полученные при $t=0, \quad 2 l / c, \quad 4 l / c, \quad 6 l / c \ldots$ Здесь тоже наблюдается знакопеременность угловых коэффициентов. Наблюдается стремление по времени к $p_{00}$. Наибольшие значения давления при $l=2000,1000,500$ м... достигается при $t=2 l / c$. 


\begin{tabular}{|c|c|c|c|c|c|c|}
\hline Impact Factor: & $\begin{array}{l}\text { ISRA (India) } \\
\text { ISI (Dubai, UAF } \\
\text { GIF (Australia) } \\
\text { JIF }\end{array}$ & $\begin{array}{l}=1.344 \\
=0.829 \\
=0.564 \\
=1.500\end{array}$ & $\begin{array}{l}\text { SIS (USA) } \\
\text { PИНЦ (Russia) } \\
\text { ESJI (KZ) } \\
\text { SJIF (Morocco) }\end{array}$ & $\begin{array}{l}=0.912 \\
=0.234 \\
=1.042 \\
=\mathbf{2 . 0 3 1}\end{array}$ & $\begin{array}{l}\text { ICV (Poland) } \\
\text { PIF (India) } \\
\text { IBI (India) }\end{array}$ & $\begin{array}{l}=6.630 \\
=1.940 \\
=4.260\end{array}$ \\
\hline
\end{tabular}

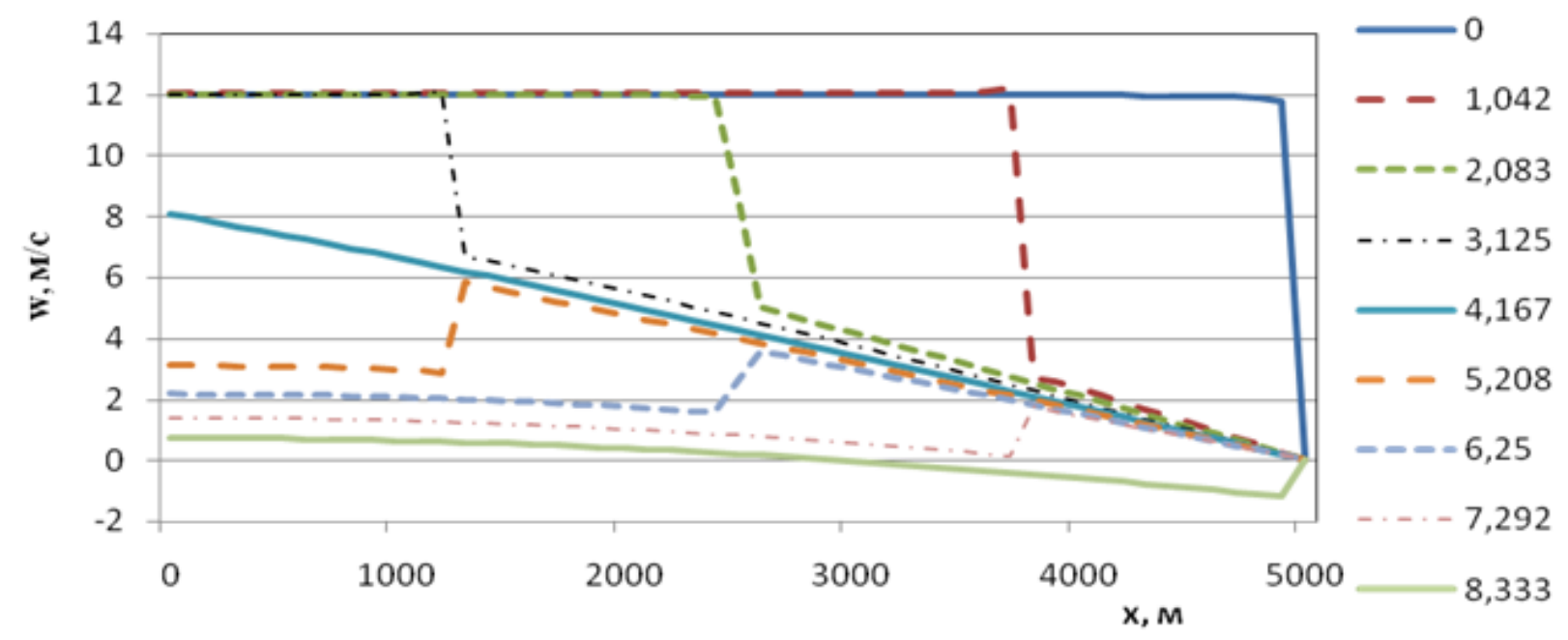

Рисунок 1 - Кривые скорости потока на участке с длиной 5000 п при шаге времени $l /(4 c)$.

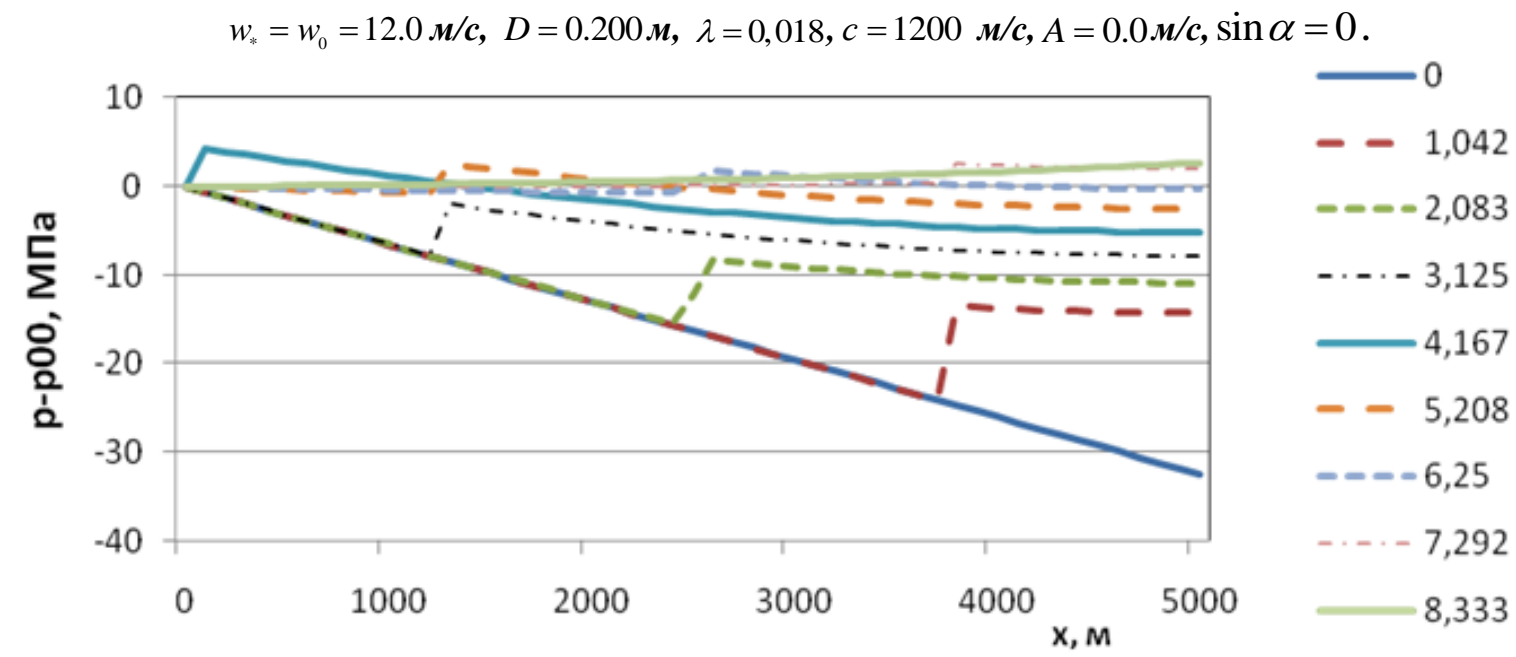

Рисунок 2 - Кривые избыточного давления $p-p 00$ в трубопроводе с длиной 5000 м, соответствующие времени с шагом $l /(4 c)$. Остальные данные см. рис. 1.

Следующая серия расчетов проводилась для различных значений параметра уклона $\sin \alpha$. Принимали $\sin \alpha=1.0,0.0,-0.5, \quad-0.66078$ и -1.0 при длине участка $l=1000$ м. Как отдельный случай рассмотрели $\sin \alpha_{к p}$ «критический» уклон, когда в обычном режиме работы трубопровода без скачков давление по длине участка остается постоянным [5]. В этом случае потеря энергии на сопротивление трения компенсируется за счет потенциальной энергии гравитации. В нашем случае получено $\sin \alpha_{K p}=-2 a w_{0} / g=-0.66078 . \quad$ Дальнейшее уменьшение уклона приводит к «постперевальному» режиму течения [3,6,7], где давление по длине возрастает.

Следует отметить, что кривые скорости, полученные при $l=1000$ м, практически взаимно идентичны - подобны кривым рис. 5.

При $\sin \alpha=1$ наибольшее значение давления ожидается при $t \rightarrow l / c$, а при $\sin \alpha=-0.5$, $\sin \alpha_{\text {Кр }}=-0.66, \quad \sin \alpha=-1-$ при $t \rightarrow 2 l / c$. В качестве примера приведены графики давления, полученные при $\sin \alpha=-0.5$ (рис. 6). В случае $\sin \alpha=\sin \alpha_{\text {Kр }}$ график исходного распределения $p(x, 0)-p_{00}$ совпадает с координатной оси абсцисс, а при $\sin \alpha=-1-$ выше чем на рис. 6 


\begin{tabular}{l|lrl|l|ll} 
& ISRA (India) & $=\mathbf{1 . 3 4 4}$ & SIS (USA) & $=\mathbf{0 . 9 1 2}$ & ICV (Poland) & $=\mathbf{6 . 6 3 0}$ \\
Impact Factor: & ISI (Dubai, UAE) $=\mathbf{0 . 8 2 9}$ & PUHU (Russia) $=\mathbf{0 . 2 3 4}$ & PIF (India) & $=\mathbf{1 . 9 4 0}$ \\
& GIF (Australia) & $\mathbf{0 . 5 6 4}$ & ESJI (KZ) & $=\mathbf{1 . 0 4 2}$ & IBI (India) & $=\mathbf{4 . 2 6 0}$
\end{tabular}
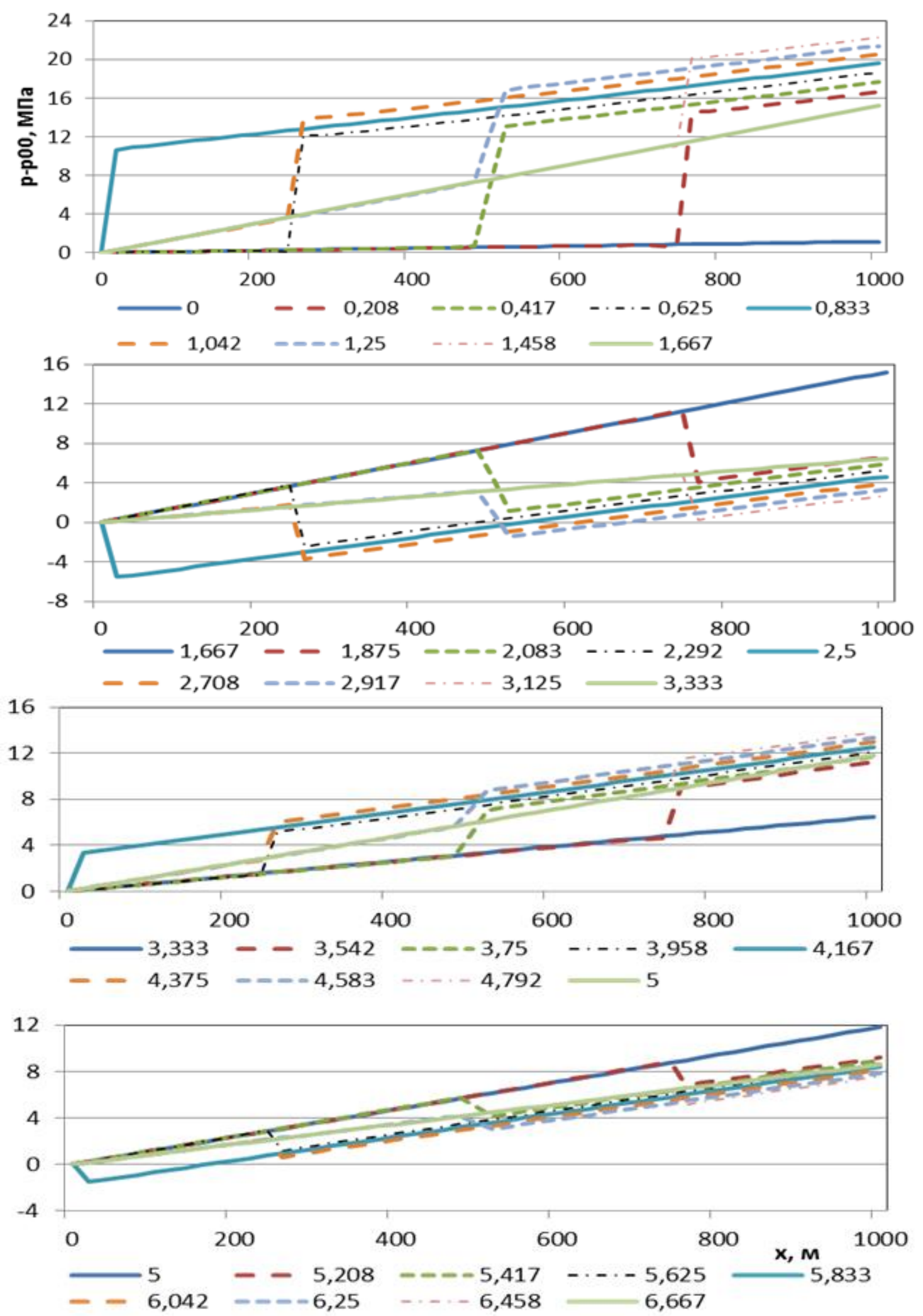

Рисунок 3 - Кривые давления после остановки потока направленного вертикально вниз ( $\sin \alpha=-1.0$ ) на расстоянии 1000 м. Остальные данные см. рис. 1. 


\begin{tabular}{|c|c|c|c|c|c|c|}
\hline Impact Factor: & $\begin{array}{l}\text { ISRA (India) } \\
\text { ISI (Dubai, UAF } \\
\text { GIF (Australia) } \\
\text { JIF }\end{array}$ & $\begin{array}{r}=1.344 \\
=0.829 \\
=0.564 \\
=1.500\end{array}$ & $\begin{array}{l}\text { SIS (USA) } \\
\text { PИНЦ (Russia) } \\
\text { ESJI (KZ) } \\
\text { SJIF (Morocco) }\end{array}$ & $\begin{array}{l}=0.912 \\
=0.234 \\
=1.042 \\
=2.031\end{array}$ & $\begin{array}{l}\text { ICV (Poland) } \\
\text { PIF (India) } \\
\text { IBI (India) }\end{array}$ & $\begin{array}{l}=6.630 \\
=1.940 \\
=4.260\end{array}$ \\
\hline
\end{tabular}
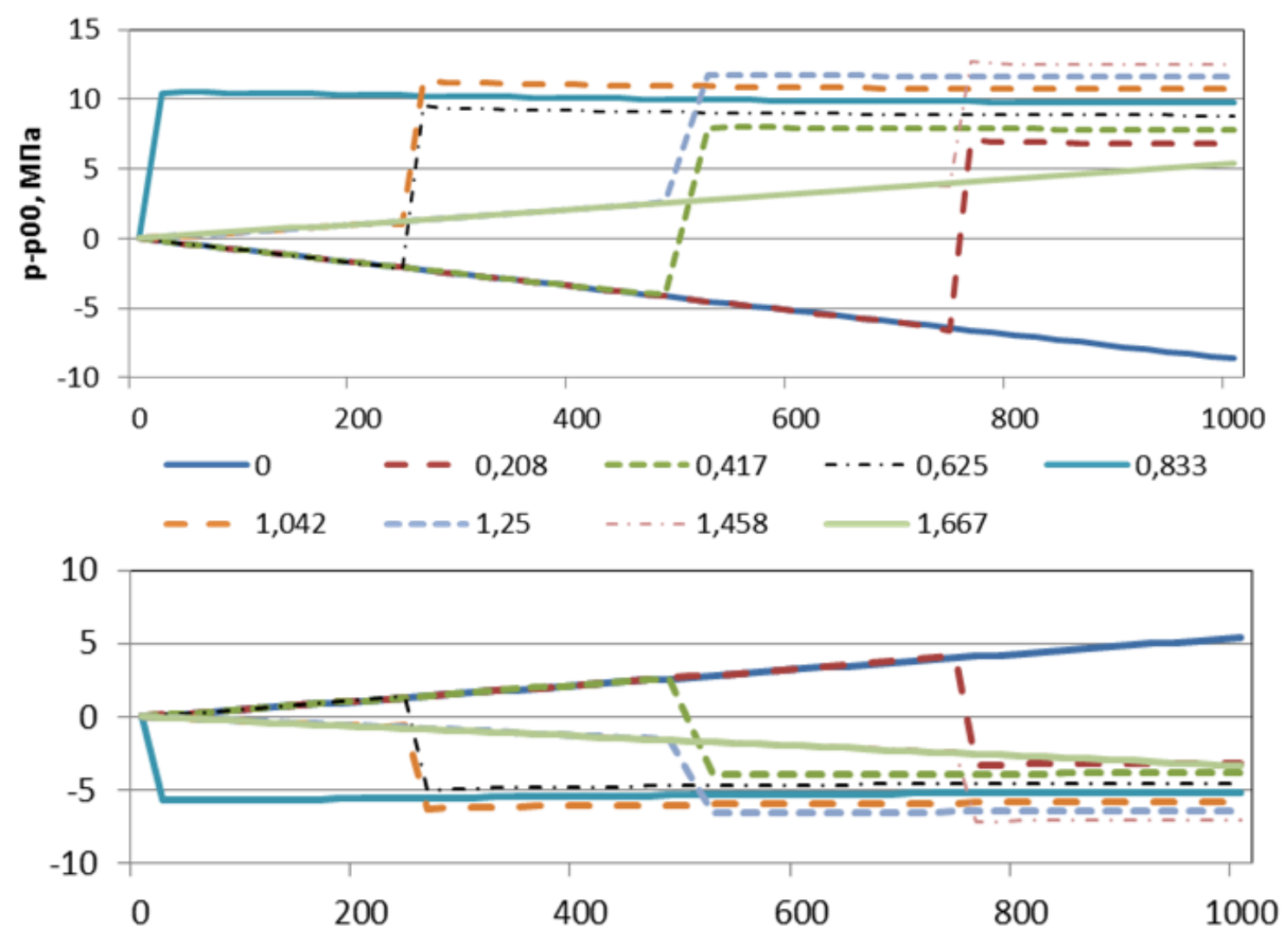

$$
\begin{aligned}
& \longrightarrow 1,667--1,875---2,083-\cdot-2,292-2,5 \\
& -2,708----2,917-\cdots-3,125-3,333
\end{aligned}
$$
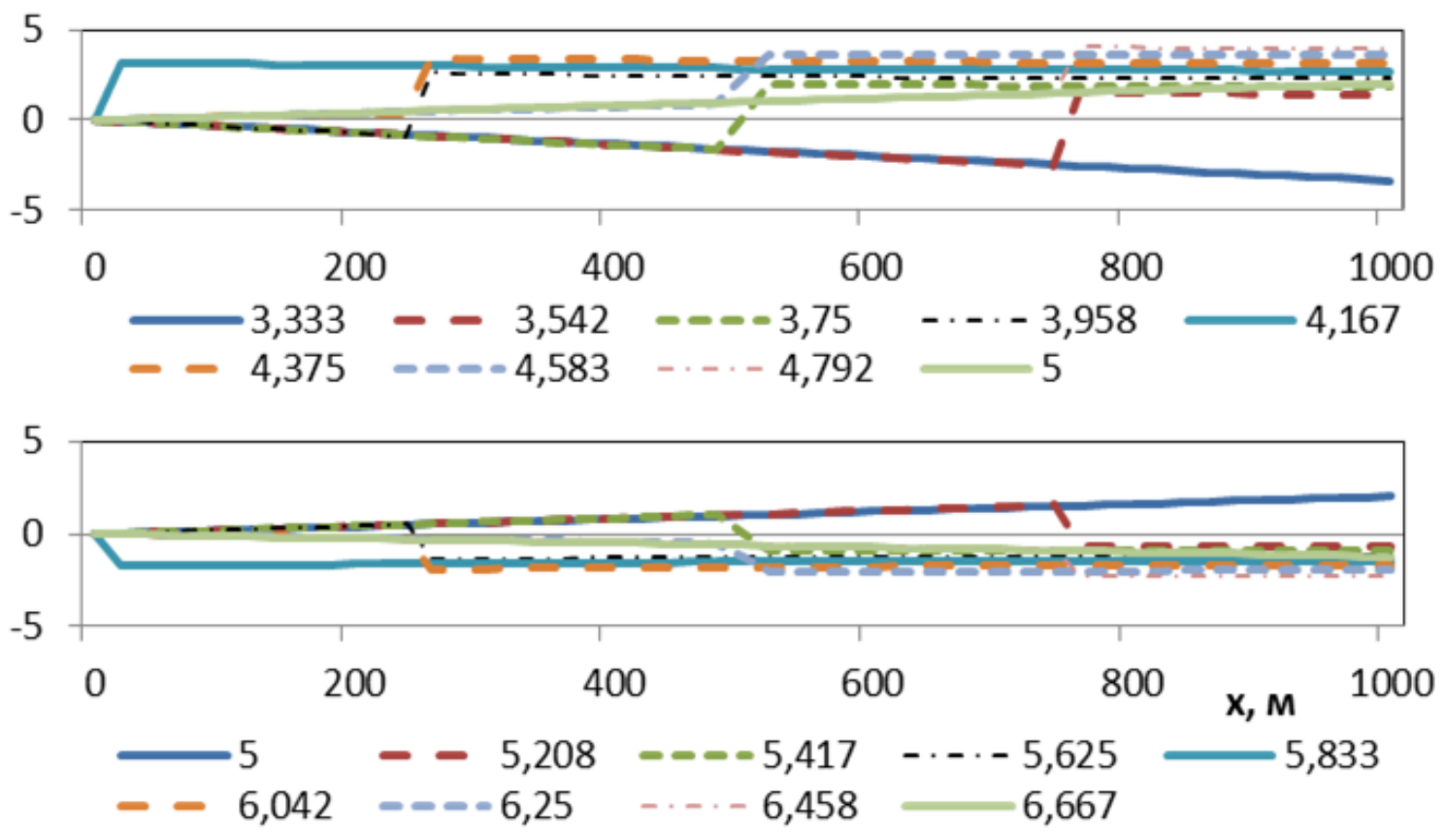

Рисунок 4 - Кривые статического давления в горизонтальном трубопроводе с длиной 1000 м, соответствующие моментам времени с шагом $l /(4 c)$. Остальные данные см. рис. 1. 


\begin{tabular}{l|lrl|l|ll} 
& ISRA (India) & $=\mathbf{1 . 3 4 4}$ & SIS (USA) & $=\mathbf{0 . 9 1 2}$ & ICV (Poland) & $=\mathbf{6 . 6 3 0}$ \\
Impact Factor: & ISI (Dubai, UAE) $=\mathbf{0 . 8 2 9}$ & PUHU (Russia) $=\mathbf{0 . 2 3 4}$ & PIF (India) & $=\mathbf{1 . 9 4 0}$ \\
& GIF (Australia) & $\mathbf{0 . 5 6 4}$ & ESJI (KZ) & $=\mathbf{1 . 0 4 2}$ & IBI (India) & $=\mathbf{4 . 2 6 0}$
\end{tabular}
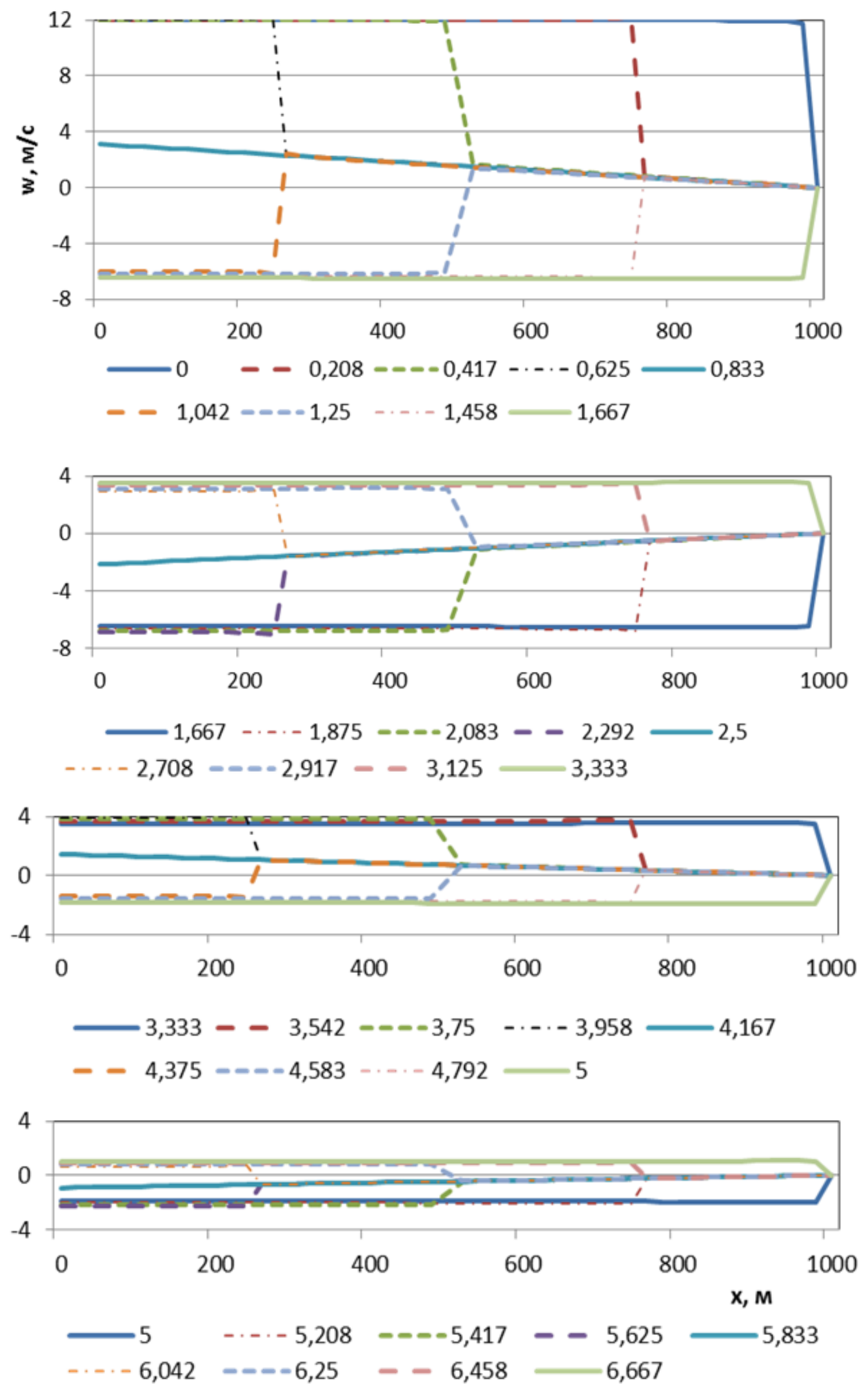

Рисунок 5 - Кривые скорости потока при различных моментах времени после остановки потока в сечении 1000 м в горизонтальном трубопроводе. Остальные данные см. рис. 1. 


\begin{tabular}{|c|c|c|c|c|c|c|}
\hline Impact Factor: & $\begin{array}{l}\text { ISRA (India) } \\
\text { ISI (Dubai, UAF } \\
\text { GIF (Australia) } \\
\text { JIF }\end{array}$ & $\begin{array}{r}=1.344 \\
=0.829 \\
=0.564 \\
=1.500\end{array}$ & $\begin{array}{l}\text { SIS (USA) } \\
\text { PИHЦ (Russia) } \\
\text { ESJI (KZ) } \\
\text { SJIF (Morocco) }\end{array}$ & $\begin{array}{l}=0.912 \\
=0.234 \\
=1.042 \\
=\mathbf{2 . 0 3 1}\end{array}$ & $\begin{array}{l}\text { ICV (Poland) } \\
\text { PIF (India) } \\
\text { IBI (India) }\end{array}$ & $\begin{array}{l}=6.630 \\
=1.940 \\
=4.260\end{array}$ \\
\hline
\end{tabular}
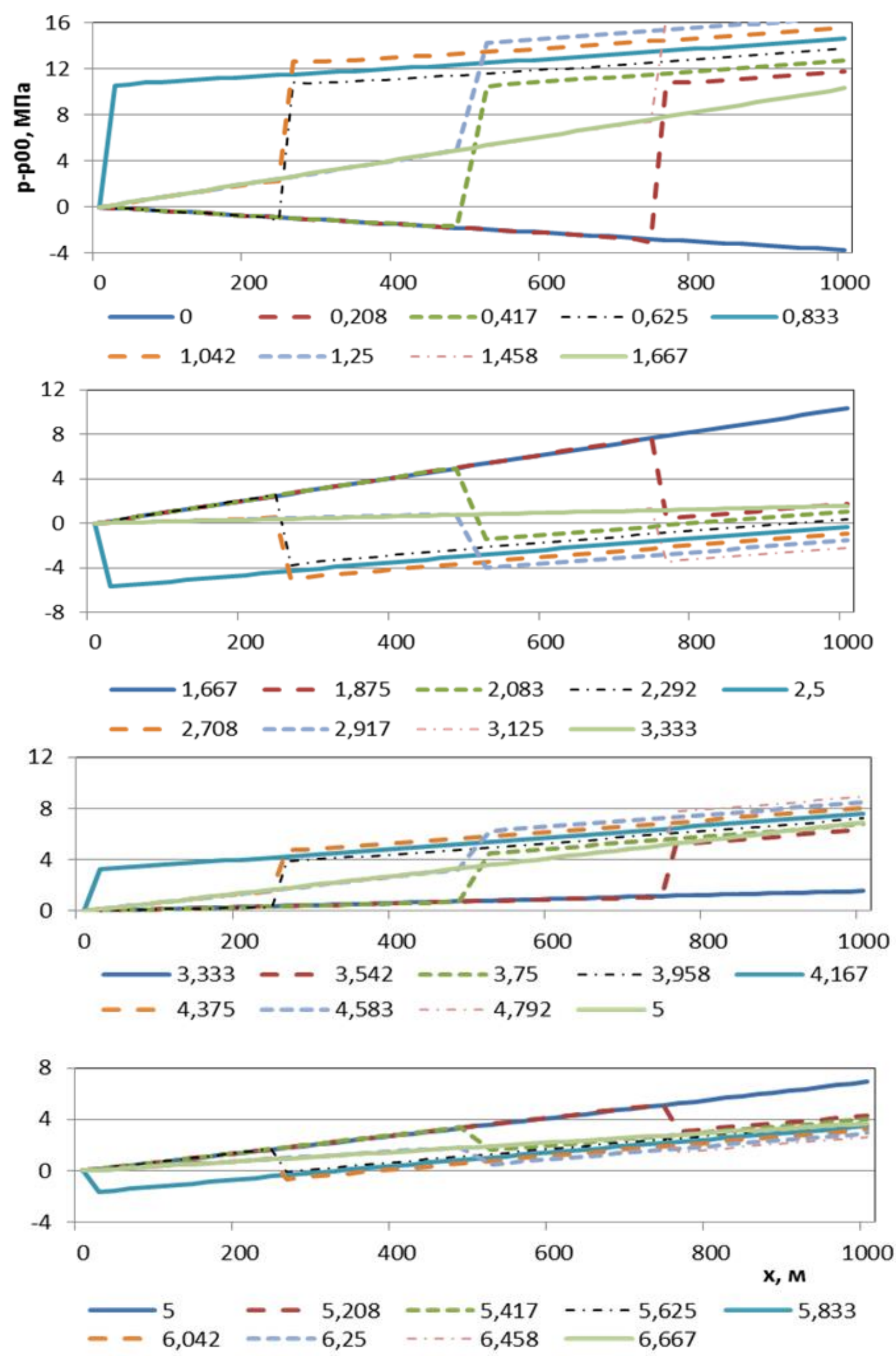

Рисунок 6 - Кривые статического давления в трубопроводе с $\sin \alpha=-0.5$ при торможении жидкости в сечении 1000 м, построенные с шагом времени $l /(4 c)$. Остальные данные см. рис. 1. 


\begin{tabular}{|c|c|c|c|c|c|c|}
\hline Impact Factor: & $\begin{array}{l}\text { ISRA (India) } \\
\text { ISI (Dubai, UAF } \\
\text { GIF (Australia) } \\
\text { JIF }\end{array}$ & $\begin{array}{l}=1.344 \\
=0.829 \\
=0.564 \\
=1.500\end{array}$ & $\begin{array}{l}\text { SIS (USA) } \\
\text { PИНЦ (Russia) } \\
\text { ESJI (KZ) } \\
\text { SJIF (Morocco) }\end{array}$ & $\begin{array}{l}=0.912 \\
=0.234 \\
=1.042 \\
=2.031\end{array}$ & $\begin{array}{l}\text { ICV (Poland) } \\
\text { PIF (India) } \\
\text { IBI (India) }\end{array}$ & $\begin{array}{l}=6.630 \\
=1.940 \\
=4.260\end{array}$ \\
\hline
\end{tabular}

Обсуждение и выводы. Сформулирована и решена задача о торможении жидкости в наклонном трубопроводе, функционирующем в стационарном режиме. Переход к однородным граничным условиям позволил определить собственных функций по $x$ для скорости и давления соответственно в виде $X_{n}(x)=\sin \frac{2 n-1}{2}$ и $Y_{n}(x)=\sin \frac{2 n-1}{2} \frac{\pi x}{l}$.

В зависимости от значения

дискриминанта

$\mathfrak{D}_{n}=\left(\frac{\lambda w_{0}}{2 D}\right)^{2}-\left(\frac{2 n-1}{2} \frac{\pi c}{l}\right)^{2}$

характеристического

уравнения собственные функции $T_{n}(t)$ задачи имели три разные решения [8]: $\begin{aligned} e^{-a t}\left(\operatorname{ch} \sqrt{\mathfrak{D}_{n}} t+\frac{a}{\sqrt{\mathfrak{D}_{n}}} \operatorname{sh} \sqrt{\mathfrak{D}_{n}} t\right) & \text { при } & \mathfrak{D}_{n}>0, \\ e^{-a t}(1+a t) & \text { при } & \mathfrak{D}_{n}=0,\end{aligned}$ $\cos \sqrt{\left|\mathfrak{D}_{n}\right|} t+\frac{a}{\sqrt{\left|\mathfrak{D}_{n}\right|}} \sin \sqrt{\left|\mathfrak{D}_{n}\right|} t$ при $\mathfrak{D}_{n}<0$.
Результаты показали, что при больших длинах участка роль силы инерции в решениях задачи относительно скорости потока незначительна. Данное суждение подтверждает известный вывод специалистов по трубопроводному транспорту.

Выявлено, что с сокращением длины участка более заметно проявляются отражения волн уплотнения. Кривые скорости и давления при $t=n l / c$ получаются без скачка. При малых значениях $t \neq n l / c$ образуются трехзвенные кривые. При малых длинах участка $l$ кривые скорости при $t=0$ и $t=(2 n-1) l / c$ визуально составляют огибающих. А кривые давления при $t=2 n l / c$ составляют визуальных огибающих кривых давления при малых временах $[9,10]$.

Выделены точки трубопровода и времена, при которых достигаются наибольшие значения давления в наклонном трубопроводе.

\section{References:}

1. Charnyy IA (1975) Neustanovivsheesya dvizhenie real'noy zhidkosti $\mathrm{v}$ trubakh.- M.: Nedra, 1975. - 296 p.

2. Budak BM, Samarskiy AA, Tikhonov AN (1972) Sbornik zadach po matematicheskoy fiziki. - M.: Nauka, 1972. - 688 pp. (158, 252 p.)

3. Aliev RA, Belousov VD, Nemudrov AG, Yufin VA, Yakovlev AG (1988) Truboprovodnyy transport nefti i gaza. / Pod obshch. red. - 2-e izd. - M.: Nedra, 1988. - 368 p. (Pereval'naya tochka 110-111 p.).

4. Tikhonov AN, Samarskiy AA (1977) Uravneniya matematicheskoy fiziki. - M.: Nauka, 1977. -735 p.

5. Kalitkin NN (1978) Chislennye metody. - M.: Nauka, 1978. - 512 p.

6. Bobrovskiy SA, Shcherbakov SG, Guseyn-zade MA (1972) Dvizhenie gaza v gazoprovodakh s putevym otborom. - Moskva, «Nauka» - 1972. -113 p.

7. Seleznev VE, Aleshin VV, Pryalov SN (2007) Sovremennye komp'yuternye trenazhery $\mathrm{V}$ truboprovodnom transporte. - Moskva: MAKS Press. $-2007 .-200$ p.
8. Khuzhaev IK, Mamadaliev KA, Kukanova MA (2015) Analiticheskoe reshenie zadachi o rasprostranenii volny uplotneniya $\mathrm{v}$ naklonnom truboprovode, vyzvannoy tormozheniem zhidkosti // Problemy vychislitel'noy i prikladnoy matematiki. - T., 2015. - №2. - pp. 65-79.

9. Khuzhaev IK, Mamadaliev KA (2015) Zadachi prekrashcheniya otbora i zakachki gaza $\mathrm{v}$ naklonnye gazoprovody pri chastichnom uchete sily inertsii // Doklady Respublikanskoy nauchno-tekhnicheskoy konferentsii: Sovremennoe sostoyanie i perspektivy primeneniya informatsionnykh tekhnologiy $\mathrm{v}$ upravlenii, Tashkent, 7-8 sept. 2015, pp. 260266.

10. Khuzhaev IK, Mamadaliev KA (2014) O rasprostranenii volny uplotneniya po naklonnomu truboprovodu // Perspektivy effektivnogo razvitiya informatsionnykh tekhnologiy i telekommunikatsionnykh sistem: sb. dokladov Respublikanskoy nauchnotekhnicheskoy konferentsii, 13-14 march. Ch. 1. - Tashkent, 2014 - pp. 170-172. 